\title{
Présentation d'une maladie ophthalmique liée au SIDA chez un patient sévèrement immunodéprimé
}

\author{
PAR SALLY CHETRIT, OD
}

\section{Introduction}

L e virus d'immunodéficience humaine (VIH) est un rétrovirus qui cause des dommages progressifs au système immunitaire en attaquant et en détruisant de façon sélective un type particulier de globules blancs, les lymphocytes T-CD4. Le syndrome d'immunodéficience acquise (SIDA) est connu comme étant le dernier stade de l'infection à VIH ${ }^{1}$. Un patient est réputé avoir le SIDA lorsque sa numération de lymphocytes T-CD4 diminue en deçà de 200 cellules par microlitre, ou si le pourcentage de lymphocytes T-CD4 est inférieur à $14 \%$ de la masse totale de lymphocytes ${ }^{2}$. Les principaux facteurs de risque du VIH comprennent les injections de drogue et les rapports sexuels à risque élevé3. En décembre 2008, les centres de prévention et de lutte contre la maladie estimaient à 1,1 million le nombre de personnes vivant avec le VIH aux États-Unis. Ce nombre devrait augmenter étant donné que le traitement aux antirétroviraux prolonge la vie des patients infectés au VIH et accroît inévitablement les possibilités de transmission à d'autres ${ }^{4}$. Au cours des 25 dernières années, la population affectée par l'épidémie du SIDA a varié, puisque $25 \%$ des infections par VIH sont maintenant diagnostiquées chez les femmes et que le virus touche maintenant d'une façon beaucoup plus disproportionnée les minorités raciales et ethniques. Plus de la moitié des nouveaux diagnostics sont posés chez les Afro-Américains ${ }^{5,6}$.

La rétinopathie à VIH est la manifestation oculaire la plus courante du SIDA, touchant entre $40 \%$ et $60 \%$ des personnes séropositives pour le VIH dont le taux de lymphocytes T-CD4 est inférieur à 100 cellules par microlitre 7 . Sur le plan clinique, cette microvasculopathie rétinienne présente des nodules cotonneux et des microanévrismes sur le pôle postérieur. La présence de la

\section{RÉSUMÉ}

Contexte : La rétinopathie liée au virus de l'immunodéficience humaine (VIH) est la manifestation oculaire la plus courante du syndrome d'immunodéficience acquise (SIDA), servant de marqueur important chez les patients présentant un état immunitaire fortement déprimé qui sont à risque de contracter des infections oculaires opportunistes sévères qui menacent la vision. Le présent rapport de cas décrit une présentation d'une rétinopathie à VIH et d'une rétinite à cytomégalovirus (CMV) et examine les options de prise en charge actuellement utilisées.

Rapport de cas: Un homme de race noire de 40 ans se présente pour un examen complet de la vue afin d'exclure la possibilité d'une maladie ophtalmique liée au VIH. À ses premières visites, un examen du fond de l'œil à pupilles dilatées révèle une rétinopathie au VIH à I'œil gauche. Après d'autres examens et du fait que sa capacité immunitaire diminuait, le patient contracte une rétinite à CMV dans I'œil droit. On administre avec succès au patient un traitement antirétroviral hautement actif (HAART) et du valgancyclovir par voie orale.

Conclusion : La rétinite à CMV est l'infection oculaire opportuniste la plus courante associée au SIDA. Un traitement systémique HAART et une thérapie concomitante contre le CMV permettent de reconstituer le système immunitaire et de faire régresser la rétinite.

Mots clés : virus d'immunodéficience humaine $(\mathrm{VIH})$; syndrome d'immunodéficience acquise (SIDA), rétinopathie à VIH; rétinite à cytomégalovirus; gancyclovir; traitement antirétroviral hautement actif (HAART). 


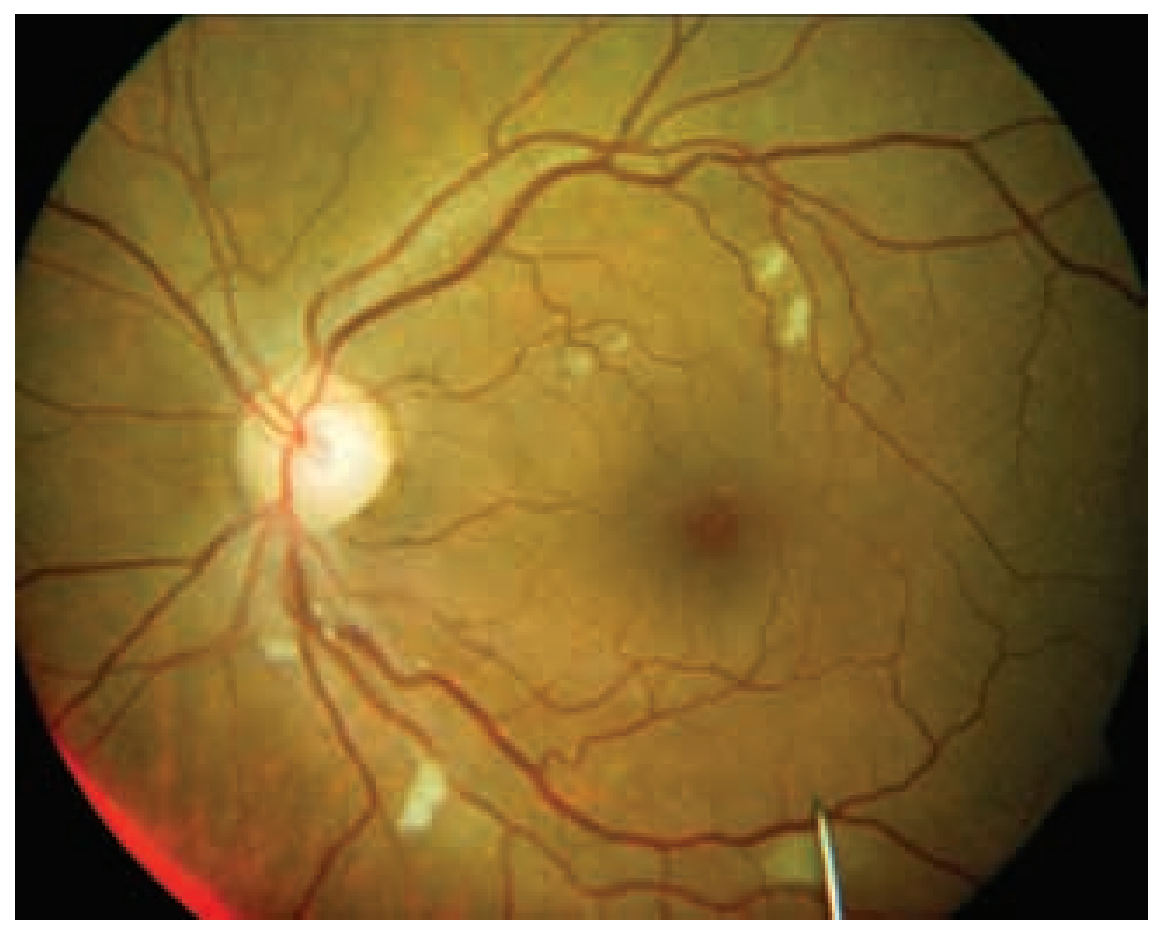

Figure 1: Multiples nodules cotonneux sur le pôle postérieur, ail gauche, première visite.

rétinopathie à VIH est un important marqueur chez les patients présentant un état immunitaire fortement déprimé qui sont à risque de contracter des infections oculaires opportunistes sévères menaçant la vision ${ }^{7}$.

La rétinite à cytomégalovirus (RCMV) est l'infection oculaire opportuniste la plus courante associée au SIDA, puisqu'elle affecte environ $9 \%$ des patients sidatiques $^{7}$. On trouve deux formes cliniques de la RCMV. La forme fulminante présente une nécrose rétinienne confluente et des hémorragies dans la région du pôle postérieur. La forme indolente de la RCMV prend l'aspect de lésions granulaires dans la rétine périphérique, avec peu ou pas d'hémorragies. Le traitement comprend typiquement une thérapeutique antirétrovirale hautement active (HAART) avec administration concomitante d'agents antiCMV particuliers ${ }^{7}$.

Voici le cas d'un patient séropositif gravement immunodéprimé qui a contracté une rétinopathie à VIH et par la suite une RCMV.

\section{Rapport de cas}

Un homme de race noire de 40 ans hospitalisé et séropositif pour le VIH est dirigé à la clinique oculaire du Woodhull Medical and Mental Health Center (Brooklyn, New York) pour un examen complet de la vue afin d'exclure toute possibilité de maladie ophtalmique associée au VIH.

Le patient reçoit un diagnostic de VIH en juillet 2008. On le perd de vue jusqu'à son admission, douze semaines plus tard, à l'hôpital où il présente des signes et symptômes d'une œsophagite aiguë. On le dirige vers la clinique oculaire pour sa première évaluation. À son arrivée, il ne présente aucun symptôme oculaire, et un examen des systèmes ne révèle qu'une séropositivité concomitante à une œsophagite.

Au moment de son admission, le patient présente une vitesse de sédimentation élevée de $82 \mathrm{~mm} / \mathrm{h}$ (valeur de référence : 0-15 mm/h), des titres positifs d'immunoglobuline $\mathrm{G}$ au cytomégalovirus, un test positif de dépistage des anticorps du VIH (transfert Western), une quantité élevée d'ADN du CMV, avec 2733 copies/mL (valeur de référence : $<200$ copies $/ \mathrm{mL}$ ) et un taux de lymphocytes T-CD4 de 7 cellules/ $\mu \mathrm{L}$ (valeur de référence : 544-1 894 cellules/ $\mu \mathrm{L})$.

D'abord traité par HAART, le patient affiche un taux d'observance supérieur à $95 \%$. La médication du patient comprend les agents suivants : Kaletra ${ }^{\circledR}$ (Laboratoires Abbott, North Chicago, Illinois) qui est une 


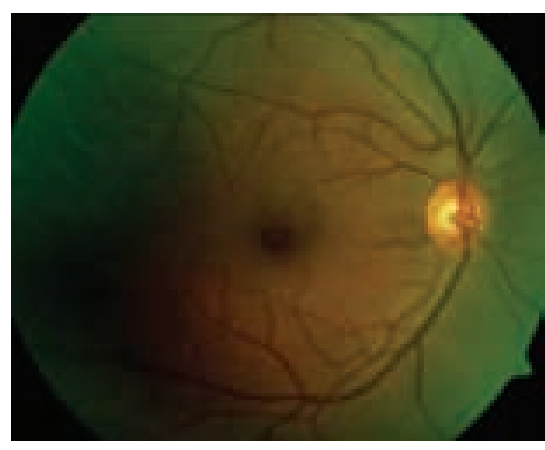

Figure 2 : Pôle postérieur sans aucun signe de rétinopathie, ail droit, première visite.

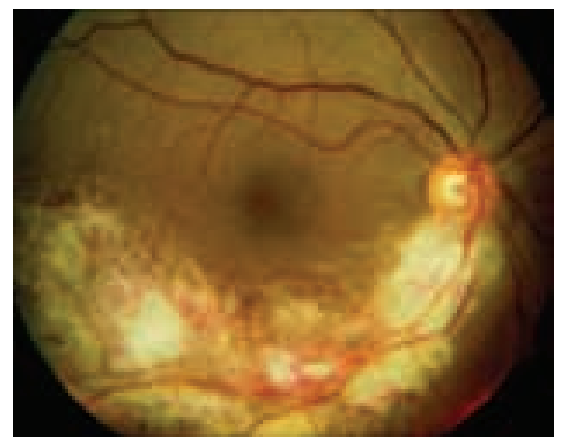

Figure 3 : Rétinite à $C M V$, xil droit : hémorragie rétinienne, nécrose et adème, suivi après trois mois.

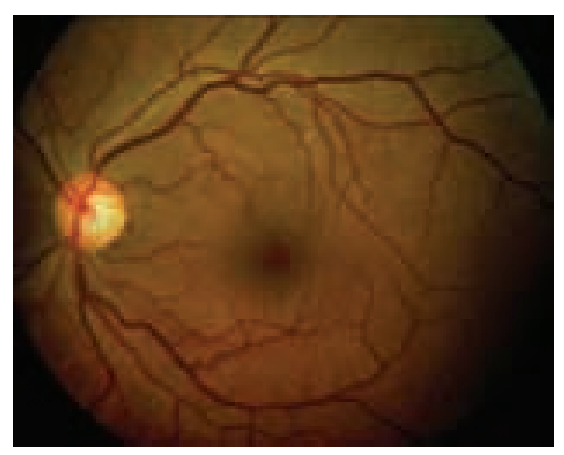

Figure 4 : Nodule cotonneux en résorption, ail gauche, suivi après trois mois. coformulation de deux inhibiteurs de la protéase : lopinavir et ritonavir, et Truvada ${ }^{\circledR}$ (Gilead Sciences Inc, Foster City, Californie), une combinaison de deux inhibiteurs nucléosidiques de la transcriptase inverse, à savoir emtricitabine et ténofovir. Les deux médicaments sont administrés par voie orale deux fois par jour.

Le premier examen oculaire révèle de multiples nodules cotonneux dans le pôle postérieur de l'œil gauche sans aucun signe d'hémorragie ou d'exsudation (figure 1). L'œil droit semble n'avoir aucune anomalie microvasculaire (figure 2). L'acuité visuelle mesurée est de 20/20- dans l'œil droit et l'œil gauche. Le patient reçoit un diagnostic de rétinopathie liée au VIH dans l'œil gauche. Aucun traitement supplémentaire n'est indiqué, cependant étant donné le taux très bas de $\mathrm{CD} 4$, on lui demande de poursuivre la thérapie HAART et de revenir à la clinique oculaire un mois plus tard pour un suivi. Outre les nodules cotonneux décelés à la première visite, on n'observe aucune trace de RCMV. Comme son taux d'ADN de CMV est élevé, on conclut qu'il a un risque élevé de contracter cette affection.

Après un mois, son taux de CD4 s'améliore légèrement (10,4 cellules/ $\mu \mathrm{L})$. L'acuité visuelle demeure stable à 20/20- dans l'œil droit et dans l'œil gauche. Un examen du fond d'œil à pupilles dilatées révèle deux nodules cotonneux sur le pôle postérieur de l'œil gauche. L'état de l'œil droit ne semble pas bouger depuis la visite précédente et on ne note aucun signe de rétinopathie ou de rétinite. On poursuit donc le plan de diagnostic et de prise en charge et on demande au patient de revenir le mois suivant pour un suivi.

Un mois plus tard, l'examen du fond d'œil à pupilles dilatées révèle une stabilité de l'acuité visuelle, cependant on décèle un nodule cotonneux sur le pôle postérieur de l'œil droit, accompagné d'une petite hémorragie. On constate aussi deux nodules cotonneux sur le pôle postérieur de l'œil gauche. On diagnostique alors chez le patient une rétinopathie à VIH dans les deux yeux, très susceptible de déboucher sur une RCMV. On lui demande de revenir à la clinique deux semaines plus tard pour un autre suivi.

Le patient se présente un mois plus tard. Le taux de CD4 à ce moment-là est stable à 10 cellules $/ \mu \mathrm{L}$. Le patient dit poursuivre fidèlement le traitement HAART. On constate une baisse de l'acuité visuelle de l'œil droit à 20/40-, alors que l'acuité visuelle de l'œil gauche est relativement stable à 20/20-. Un examen à pupilles dilatées révèle dans l'œil droit une hémorragie rétinienne, une nécrose et un œdème le long de l'arcade

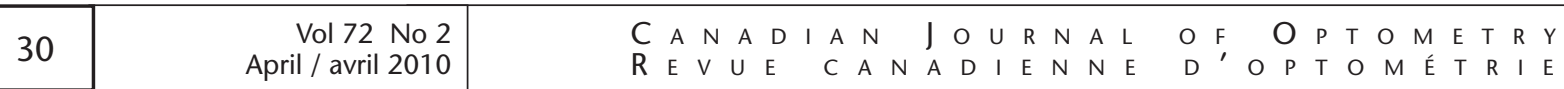


TABLEAU 1.

Corrélation entre l'état immunitaire du patient et les résultats ophtalmiques

\begin{tabular}{|c|c|c|c|c|c|c|}
\hline Visite & Date & $\begin{array}{l}\text { Taux de } \\
\text { CD4+ } \\
\text { (cell./ } \mu \mathrm{L})\end{array}$ & $\begin{array}{l}\text { Charge viral } \\
\text { CMV } \\
\text { (copies } / \mathrm{mL} \text { ) }\end{array}$ & $\begin{array}{c}\text { Conclusions } \\
\text { ophtalmiques } \\
\text { d'examen du fond } \\
\text { d'œil O.D. }\end{array}$ & $\begin{array}{c}\text { Conclusions } \\
\text { ophtalmiques } \\
\text { d'examen du fond } \\
\text { d'œil O.S. }\end{array}$ & Diagnostic \\
\hline 1 & $10 / 2008$ & 9 & 2733 & Normal & $\begin{array}{l}\text { Multiples nodules } \\
\text { cotonneux sur } \\
\text { le pôle postérieur. } \\
\text { Aucune hémorragie. } \\
\text { Aucune exsudation. }\end{array}$ & $\begin{array}{c}\text { Rétinopathie à VIH } \\
\text { O.S. }\end{array}$ \\
\hline 2 & $11 / 2008$ & 10,4 & Non testé & Normal & $\begin{array}{l}\text { Deux nodules } \\
\text { cotonneux sur } \\
\text { le pôle postérieur. }\end{array}$ & $\begin{array}{c}\text { Retinopathie à VIH } \\
\text { O.S. }\end{array}$ \\
\hline 3 & $12 / 2008$ & Non testé & Non testé & $\begin{array}{l}\text { Un nodule cotonneux et } \\
\text { une petite hémorragie } \\
\text { sur le pôle postérieur. }\end{array}$ & $\begin{array}{l}\text { Deux nodules } \\
\text { cotonneux sur } \\
\text { le pôle postérieur. }\end{array}$ & $\begin{array}{c}\text { Retinopathie à VIH } \\
\text { O.S. }\end{array}$ \\
\hline 4 & $01 / 2009$ & 10 & Non testé & $\begin{array}{l}\text { Hémorragie rétinienne, } \\
\text { nécrose et œdème } \\
\text { le long de l'arcade } \\
\text { vasculaire inférieure. }\end{array}$ & $\begin{array}{c}\text { Un nodule cotonneux } \\
\text { se résorbant sur le pôle } \\
\text { postérieur }\end{array}$ & $\begin{array}{l}\text { RCMV O.D. } \\
\text { Rétinopathie à VIH } \\
\text { O.S. }\end{array}$ \\
\hline 5 & $01 / 2009^{*}$ & 10 & 252 & $\begin{array}{l}\text { Hémorragie rétinienne, } \\
\text { nécrose et œdème en } \\
\text { résorption.. }\end{array}$ & Normal & $\begin{array}{l}\text { Rétinite à CMV en } \\
\text { résorption O.D. }\end{array}$ \\
\hline 6 & 03/2009 & 9 & $<200$ & $\begin{array}{l}\text { Hémorragie rétinienne, } \\
\text { nécrose et œdème en } \\
\text { résorption.. }\end{array}$ & Normal & $\begin{array}{l}\text { Rétinite à CMV en } \\
\text { résorption O.D. }\end{array}$ \\
\hline
\end{tabular}

* Après mise en place d'une thérapie anti-CMV.

vasculaire inférieure (figure 3). Seul un nodule cotonneux en voie de résorption apparait dans l'œil gauche (figure 4). On diagnostique une RCMV dans l'œil droit et une rétinopathie à VIH dans l'œil gauche. On commence aussitôt un traitement à valgancyclovir 900 mg, deux fois par jour par voie orale pendant deux semaines. On lui demande de revenir quatre jours plus tard à la clinique.
Les examens de suivi révèlent une amélioration de la RCMV dans l'œil droit et de la rétinopathie à VIH dans l'œil gauche. Après une thérapie anti-CMV, le taux d'ADN de CMV du patient (par réaction en chaîne de la polymérase en temps réel) diminue à 252 copies $/ \mathrm{mL}$. Le patient reçoit son congé de l'hôpital environ deux semaines après le début du traitement au valgancyclovir par voie orale. Une fois prise la dose d'induction de deux semaines de valgancyclovir, le patient reçoit une dose d'entretien de valgancyclovir par voie orale, $900 \mathrm{mg}$ par jour, pour une période indéfinie.

À son plus récent suivi, environ un mois et demi après son diagnostic de RCMV, le taux de CD4 demeure peu élevé à 9 cellules/ $\mu \mathrm{L}$. On mesure l'acuité visuelle de l'œil droit à 20/25 et celle de l'œil gauche à $20 / 20$. Un examen du fond d'œil révèle une rétinite régressive dans l'œil droit et aucun signe d'une rétinopathie à VIH dans l'œil gauche. Pour cette raison, on demande au 
patient de continuer sa thérapie d'entretien au valgancyclovir de $900 \mathrm{mg}$ par jour par voie orale. À l'occasion de cette visite, on modifie la thérapie HAART en y ajoutant un inhibiteur supplémentaire de la protéase, à savoir Invirase ${ }^{\circledR}$ (Roche Pharmaceuticals, Nutley, New Jersey) afin de renforcer le système immunitaire du patient.

On demande au patient de revenir deux semaines plus tard à la clinique oculaire, mais il ne se présente pas.

(Voir le tableau 1 qui résume l'état immunitaire du patient et l'évolution de la maladie ophtalmique pendant son traitement.)

\section{Discussion}

La rétinopathie liée au VIH est la manifestation oculaire la plus courante du SIDA, puisqu'elle touche entre $40 \%$ et $60 \%$ des patients séropositifs pour le VIH qui présentent typiquement un taux de CD4 inférieur à 50 cellules/ $\mu \mathrm{L}^{7}$. Cliniquement, cette microvasculopathie rétinienne présente des nodules cotonneux et des microanévrismes sur le pôle postérieur. Des hémorragies en points et taches dispersés peuvent être présentes, bien que les nodules cotonneux soient rarement associés à des hémorragies abondantes ${ }^{7}$. Ces manifestations microvasculaires ressemblent à celles de la rétinopathie diabétique non proli- férante et seraient le résultat d'une ischémie rétinienne causée par la diminution du flux des érythrocytes dans les patients séropositifs $6,8,9,10,11$. On soupçonne que cette microvasculopathie cause un amincissement progressif de la couche des fibres nerveuses de la rétine ${ }^{12}$ et une perte d'axones dans le nerf optique ${ }^{13}$ entraînant diverses anomalies de la vision, comme une diminution de la vision des couleurs, de la sensibilité différentielle et du champ visuel $^{14,15,16}$. La plupart des patients atteints de rétinopathie liée au VIH sont asymptomatiques, bien qu'ils puissent à l'occasion développer un scotome central sous l'effet d'un gros nodule cotonneux sur la macula. Cette microvasculopathie ischémique atteindra rarement la région de la fovéa pour causer un œdème maculaire ou une maculopathie séreuse qui laissera le patient symptomatique d'une vision embrouillée ou d'une perte abrupte de vision ${ }^{7}$. Dans la plupart des cas, il n'est pas indiqué de traiter la rétinopathie liée au VIH. Une photocoagulation au laser du foyer serait indiquée dans les rares cas d'œdème maculaire. La présence d'une microvasculopathie ischémique est un marqueur important chez les patients sévèrement immunodéprimés qui sont à risque de contracter des infections oculaires opportunistes plus graves menaçant la vision. Chez les patients dont le taux de CD4 est inférieur à 50 cellules $/ \mu \mathrm{L}$, il est recommandé de procéder à un examen du fond d'œil à pupilles dilatées tous les trois mois pour dépister toute RCMV ${ }^{7}$. Dans le cas indiqué précédemment, en raison de la charge élevée du cytomégalovirus, le patient était réputé être très à risque d'une RCMV et a donc subi tous les mois un examen à pupilles dilatées.

Le cytomégalovirus est un virus de l'herpès à $\mathrm{ADN}$ double brin qui cause une rétinite nécrosante chez les hôtes immunodéficients ${ }^{17,18}$. La RCMV est l'infection oculaire opportuniste la plus courante associée au SIDA, puisqu'elle touche actuellement environ $9 \%$ des patients sidatiques ${ }^{7,19}$. Même si l'incidence de la RCMV et le risque d'un décollement de rétine associé à cette rétinite ont diminué depuis le recours à la thérapie HAART, la RCMV est la principale cause de morbidité oculaire chez les patients ayant le SIDA ${ }^{7,20}$. La RCMV survient presque exclusivement chez les patients dont le taux de CD4 est inférieur à 50 cellules $/ \mu \mathrm{L}^{6,21}$. Le taux moyen de CD4 au moment du diagnostic est de 17 cellules/ $\mu \mathrm{L}^{22}$.

On trouve deux formes cliniques du RCMV. La forme fulminante présente une nécrose rétinienne confluente et des hémorragies dans la région du pôle postérieur. Les patients atteints de cette forme présenteront typiquement des symptômes d'une diminution de l'acuité visuelle et du champ visuel. 
La forme indolente de la RCMV prend l'aspect de lésions granulaires dans la rétine périphérique et s'accompagne d'une angéite givrée «frosted branch angitis » avec peu ou pas d'hémorragies ${ }^{23}$. Les patients atteints de cette forme indolente peuvent souvent se plaindre de corps flottants, ou encore être asymptomatiques. Dans les deux cas, l'infection rétinienne est dite de pleine épaisseur et commence en périphérie pour progresser de façon centrifuge le long de la vasculature rétinienne ${ }^{20}$. Même si, dans le cas précédemment mentionné, le patient était asymptomatique, des signes cliniques d'hémorragie et de nécrose sur le pôle postérieur indiquaient une infection rétinienne fulminante. Un taux peu élevé de $\mathrm{CD} 4$ produit une réaction inflammatoire, comme une hyalite, un phénomène peu courant ${ }^{24}$. Environ $15 \%$ des patients ayant une RCMV active sont asymptomatiques; voilà pourquoi on recommande de procéder à un examen trimestriel du fond d'œil à pupilles dilatées lorsque le taux de CD4 est inférieur à 50 cellules $/ \mu \mathrm{L}^{25}$.

Le traitement de la RCMV ne repose pas seulement sur l'état immunitaire du patient, mais tient aussi compte de l'emplacement de la rétinite active ${ }^{7}$. Le traitement comprend typiquement une thérapie HAART initiale ou modifiée afin de reconstituer le système immunitaire. Puisque la reconstitution immunitaire peut prendre plus de temps après un traitement HAART, il est indiqué d'utiliser de façon concomitante des agents anti-CMV chez les patients dont la RCMV sur le pôle postérieur représente une menace imminente pour la vision? ${ }^{7}$.

On traite habituellement la rétinite à CMV par administration systémique de gancyclovir ou de son promédicament, le valgancyclovir. Le gancyclovir systémique s'administre par voie intraveineuse pendant une période de deux à trois semaines, deux fois par jour, à une dose de $5 \mathrm{mg} / \mathrm{kg}$. Cette thérapie d'induction est ensuite entretenue par une dose orale quotidienne de $30 \mathrm{mg}$ de gancyclovir ${ }^{7}$. Le valgancyclovir systémique est considéré comme l'équivalent du gancyclovir dans la thérapie initiale de la RCMV, mais il a aussi l'avantage de pouvoir être administré par voie orale tant pour une thérapie d'induction que d'entretien ${ }^{6,26,27}$. Pour une thérapie d'induction, la dose orale recommandée est de $900 \mathrm{mg}$, deux fois par jour, pendant deux à trois semaines. On la fait suivre par une thérapie d'entretien orale de $900 \mathrm{mg}$, une fois par jour. Toutes ces doses supposent une fonction hépatique normale; à mesure que la clearance glomérulaire diminue, les doses d'induction et d'entretien sont également réduites. Parmi les effets secondaires courants des médicaments susmentionnés, mentionnons une dyscrasie sanguine (neutropénie, thrombocytopénie, anémie) et une néphrotoxicitée 28 . Pour tous les patients ainsi traités, on recommande une surveillance périodique de la fonction hépatique et des numérations globulaires.

Parmi les autres traitements de la RCMV, notons foscarnet, cidofovir et fomvirsen. Le foscarnet est une thérapie d'induction et d'entretien administrée par voie intraveineuse. Ce médicament est aussi efficace que le gancyclovir, sauf que ses effets secondaires intolérables sont plus nombreux ${ }^{29}$. Parmi les effets secondaires du foscarnet, mentionnons les attaques attribuables à un déséquilibre électrolytique, une neutropénie et une néphrotixicité30,31. On sait également que $37 \%$ des patients traités au foscarnet développent une résistance au médicament à l'intérieur d'une période de traitement de neuf mois ${ }^{32}$. Lorsqu'il est administré par voie intraveineuse, le cidofovir peut être combiné avec le gancyclovir oral pour un effet synergique qui prolonge la durée entre les rechutes ${ }^{33}$. Parmi les inconvénients du cidofovir, notons les effets secondaires suivants : protéinurie, neuropathie périphérique, hypotonie oculaire et uvéite antérieure ${ }^{34,35}$. On peut traiter une hypotonie oculaire en combinant l'ibopamine topique et le dexaméthasone. L’uvéite antérieure peut être traitée au moyen d'une thérapie topique aux 
corticostéroïdes ${ }^{36,37,38}$. Comme deuxième voie de traitement d'induction et d'entretien, le fomvirsen est administré par injection intravitréenne. On a limité son utilisation aux cas de RCMV qui sont réfractaires à un traitement plus classique, aux patients qui ne tolèrent pas les thérapies précédemment mentionnées, ou en présence de toute contre-indication aux médicaments de rechange. Les inconvénients du fomvirsen se rapportent aux effets secondaires associés aux injections intravitréennes, notamment : hémorragie vitréenne, décollement de la rétine et endophthalmie ${ }^{7}$.

L'implant intravitréen au gancyclovir peut être utilisé comme thérapie d'induction et d'entretien qui est tout à fait indiquée dans les cas d'une rétinite à CMV qui menace la macula. L'implant est inséré au niveau de la pars plana et libère une forte dose du médicament directement dans l'humeur vitrée pendant environ huit mois ${ }^{6}$. Parmi les avantages de l'implant au gancyclovir, mentionnons un apport local médicamenteux à des concentrations élevées se traduisant par une suppression du virus à son niveau le plus élevé et les plus longs intervalles entre les rechutes, outre l'absence de toute toxicité systémique $^{7,20}$. Les inconvénients comprennent des complications potentielles provenant de l'insertion de l'implant ${ }^{39,40}$, par exemple des hémorragies vitréennes, une endophthalmie, un décollement de la rétine et l'absence d'une protection contre un CMV systémique ou une rétinite à CMV dans l'œil contralatéral ${ }^{7}$. Dans le cas mentionné précédemment, l'infection a semblé éviter la macula, de sorte que les risques d'un traitement par implant au gancyclovir dépassaient les avantages. On a donc choisi une approche plus classique, à savoir du gancyclovir par voie orale.

L'utilisation du gancyclovir par voie orale comme traitement prophylactique contre un CMV oculaire ou un CMV systémique est fort controversée. De nombreuses études ne s'entendent pas sur l'efficacité du gancyclovir prophylactique. Parmi les autres inconvénients du gancyclovir prophylactique, mentionnons la toxicité, le coût et le développement d'une résistance ${ }^{7,41,42}$. Même si le patient dans ce cas présentait un taux peu élevé de CD4, la présence d'une rétinopathie à VIH et une charge virale élevée du CMV, on n'a pas jugé bon d'administrer un traitement prophylactique pour les raisons mentionnées précédemment.

Certains prétendent que les patients traités par une thérapie HAART qui présentent un taux élevé de CD4 lors de deux mesures consécutives espacées de trois mois et dont la RCMV demeure en régression et quiescente pendant une thérapie d'entretien de plus de quatre mois peuvent interrompre sans danger une thérapie anti-CMV à condition de surveiller étroitement toute réactivation du virus ${ }^{43}$. Plus précisément, les critères de sélection des candidats convenant à une telle interruption de la thérapie d'entretien comprennent : taux de CD4 supérieur à 100 cellules/ $\mu \mathrm{L}$ pendant au mois trois mois; rétinite totalement quiescente; longs intervalles sans rechute; thérapie HAART pendant au moins 18 mois; et charge virale VIH et CMV réduite ${ }^{7}$.

\section{Conclusion}

Ce cas illustre la progression rapide d'une maladie rétinienne liée au VIH chez des patients fortement immunodéprimés. Même si l'incidence de la RCMV a diminué depuis la mise en place du traitement HAART, elle demeure quand même la principale cause de morbidité oculaire associée au SIDA. On recommande fortement aux professionnels des soins oculovisuels d'effectuer régulièrement des examens du fond d'œil à pupilles dilatées (tous les trois mois) chez tous les patients séropositifs pour le VIH qui présentent une rétinopathie à VIH ou dont le taux de CD4 est inférieur à 50 cellules $/ \mu \mathrm{L}$. 


\section{Références}

1. Biswas J, R Fogla, L Gopal et al. Current approaches to diagnosis and management of ocular lesions in human immunodeficiency positive patients, Indian J Ophthalmol 2002, 50(2), p. 83-96.

2. US Department of Health and Human Services. MMWR Recommendations and Reports 2008. Revised surveillance case definitions for HIV infection among adults, adolescents, and children ages $<18$ months and for HIV infection and AIDS among children aged 18 months to $<13$ years, Washington (DC), US Govt Printing Office, décembre 2008.

3. US Department of Health and Human Services. HIV/AIDS in the United States. Center for Disease Control HIV/ AIDS Facts, Washington (DC), US Govt Printing Office, août 2008.

4. US Department of Health and Human Services. HIV Transmission Rates in the United States. Center for Disease Control HIV/AIDS Facts, Washington (DC), US Govt Printing Office, décembre 2008.

5. Programme commun des Nations Unies sur le VIH-SIDA. Amérique du Nord, Europe occidentale et centrale. Tendances épidémiologiques récentes. Genève, Suisse. Disponible en ligne à http://data.unaids.org/pub/ GlobalReport/2008/20080715_fs_ nawce_fr.pdf.

6. Holland GN. AIDS and ophthalmology: the first quarter century, Am J Ophthalmol, 2008, no 145 , p. 397-408.

7. Vrabec, TR. Posterior segment manifestations of HIV/AIDS, Surv Ophthalmol, 2004, no 49, p. 131-157.

8. Engstrom RE, GN Holland, WD Hardy, HJ Meiselman. Hemorheologic abnormalities in patients with human immunodeficiency virus infection and ophthalmic microvasculopathy, Am J Ophthalmol, 1990, nº 109, p. 153-161.

9. Faber DW, CA Wiley, GB Lynn et al. Role of HIV and CMV in the pathogenesis of retinitis and retinal vasculopathy in AIDS patients, Invest Ophthalmol Vis Sci, 1992, n 33, 2345.

10. Freeman WR, A Chen, DE Henderly et al. Prevalence and significance of acquired immunodeficiency syndromerelated retinal microvasculopathy, Am J Ophthalmol, 1989, n 107, p. 229-235.

11. Tufail A, GN Holland, TC Fisher et al. Increased polymorphonuclear leukocyte rigidity in $\mathrm{HIV}$ infected individuals, $\mathrm{Br} \mathrm{J}$ Ophthalmol, 2000, no 84, p. 727-731.

12. Plummer DJ, DU Bartsch, SP Azen et al. Retinal nerve fiber layer evaluation in human immunodeficiency virus-positive patients, Am J Ophthalmol, 2001, no 131, p. 216-222.

13. Tenhula WN, SZ Xu, MC Madigan et al. Morphometric comparisons of optic nerve axon loss in acquired immunodeficiency syndrome, Am J Ophthalmol, 1992, no 113, p. 14-20.

14. Quinceno JI, E Capparelli, AA Sadun et al. Visual dysfunction without retinitis in patients with acquired immunodeficiency syndrome, Am J Ophthalmol, 1992, no 113, p. 8-13.

15. Shah KH, GN Holland, F Yu et al. Contrast sensitivity and color vision in HIV-infected individuals without infectious retinopathy, Am J Ophthalmol, 2006, n 142, p. 284-292.

16. FreemanWR, ML van Natta, DA Jabs et al. Vision function in HIV-infected individuals without retinitis: report of the Studies of Ocular Complications of AIDS Research Group, Am J Ophthalmol, 2008, no 145, p. 453-462.

17. Naraqi S. Cytomegaloviruses, dans Belshe RB, rédacteur, Textbook of Human Virology, Littleton (MA), PSG Publishing Company, 1984, p. 887-927.

18. Gallant JE, RD Moore, DD Richman et al. Incidence and natural history of cytomegalovirus disease in patients with advanced human immunodeficiency virus disease treated with zidovudine, J Infect Dis, 1992, no 166, p. 1223-1227.

19. Mayo GL, et MJ Tolentino. Cytomegalovirus retinitis, Focal Points, $2007, n^{\circ} 25$, p. $1-16$.
20. Goldberg DE, LM Smithen, A Angelilli, WR Freeman. HIV-associated retinopathy in the HAART era, Retina, $2005, n^{\circ} 25$, p. 633-649.

21. Kuppermann, BD, JG Petty, DD Richman et al. Correlation between CD4+ counts and prevalence of cytomegalovirus retinitis and human immunodeficiency virusrelated noninfectious retinal vasculopathy in patients with acquired immunodeficiency syndrome, Am J Ophthalmol, 1993, no 115, p. 575-82.

22. Pertel P, R Hirschtick, J Phair et al. Risk of developing cytomegalovirus retinitis in persons infected with human immunodeficiency virus, J Acquir Immune Defic Syndr Hum Retrovirol, 1992, no 5, p. 1069-1074.

23. Raina J, JW Bainbridge, SM Shah. Decreased visual acuity in patients with cytomegalovirus retinitis and AIDS, Eye, $2000, n^{\circ} 14$, p. 8-12.

24. Palestine AG, MM Rodrigues, AM Macher et al. Ophthalmic involvement in acquired immunodeficiency syndrome, Ophthalmology, 1984, n 91, p. 1092-1099.

25. Baldassano VF, JP Dunn, J. Feinberg et al. Cytomegalovirus retinitis and low CD4+ T-lymphocyte counts, $\mathrm{N}$ Engl J Med, 1995, n 333, p. 670.

26. Curran M, S. Noble. Valgancyclovir, Drugs, 2001, no 61, p. 1145-1150.

27. Martin DF, J Sierra-Madero, S Walmsley et al. A controlled trial of valgancyclovir as induction therapy for cytomegalovirus retinitis, N Engl J Med, 2002, n 346 , p. $1119-1126$.

28. Lalezari J, J Lindley, S Walmsley et al. Roche Valgancyclovir Study Group: A safety study of oral valgancyclovir maintenance treatment of cytomegalovirus retinitis, $\mathrm{J}$ Acquir Immune Defic Syndr, 2002, n 30, p. 392-400. 
29. Studies of Ocular Complications of AIDS Research Group in collaboration with the AIDS Clinical Trials Group. Foscarnet-Gancyclovir cytomegalovirus retinitis trial 4: visual outcomes, Ophthalmology, 1994, n 101, p. 12501261.

30. Palestine AG, MA Polis, MD De Smet et al. A randomized controlled trial of foscarnet in the treatment of cytomegalovirus retinitis in patients with AIDS, Ann Intern Med, 1991, n 115 , p. 665-673.

31. Lehoang Rd, B Girard, M Robinet et al. Foscarnet in the treatment of cytomegalovirus retinitis in acquired immune deficiency syndrome, Ophthalmology, 1989, n 96, p. 865-873.

32. Jabs DA, C Enger, M Forman, JP Dunn. The Cytomegalovirus Retinitis and Viral Resistance Study Group: Incidence of foscarnet resistance and cidofovir resistance in patients treated for cytomegalovirus retinitis, Antimicrob Agents Chemother, 1998, no 42, p. 22402244.

33. Jacobson MA, S Wilson, H Stanley et al. Phase I study of combination therapy with intravenous cidofovir and oral gancyclovir for cytomegalovirus retinitis in patients with AIDS, Clin Infect Dis, $1999, n^{\circ} 28$, p. $528-533$.

34. Ambati J, KB Wynne, MC Angerame et MR Robinson. Anterior uveitis associated with intravenous cidofovir use in patients with cytomegalovirus retinitis, Br J Ophthalmol, 1999, no 83, p. 1153-1158.

35. Cochereau I, S Doan, MC Diraison et al. Uveitis in patients treated with intravenous cidofovir, Ocul Immunol Inflamm, 1999, no 7 , p. 271-272.

36. Studies of Ocular Complications of AIDS Research Group in Collaboration with the AIDS Clinical Trials Group. Parenteral cidofovir for cytomegalovirus retinitis in patients with AIDS: the HPMPC peripheral cytomegalovirus retinitis trial. A randomized, controlled trial, Ann Internal Med, 1997, n ${ }^{\circ}$ 126, p. 264-274.
37. Bainbridge JW, J Raina, SM Shah et al. Ocular complications of intravenous cidofovir for cytomegalovirus retinitis in patients with AIDS, Eye, 1999, n 13 , p. 353-356.

38. Studies of Ocular Complications of AIDS Research Group in Collaboration with the AIDS Clinical Trials Group. Longterm follow-up of patients with AIDS treated with parenteral cidofovir for cytomegalovirus retinitis: the HPMPC peripheral cytomegalovirus retinitis trial, AIDS, 2000, no 14 , p. 1571 1581.

39. Guembel HO, S Krieglsteiner, C Rosenkranz et al. Complications after implantation of intraocular devices in patients with cytomegalovirus retinitis, Graefes Arch Clin Exp Ophthalmol, $1999, n^{\circ} 237$, p. 824-829.

40. Lim JI, RA Wolitz, AH Dowling et al. Visual and anatomic outcomes associated with posterior segment complications after gancyclovir implant procedures in patients with AIDS and cytomegalovirus retinitis, Am J Ophthalmol, 1999, n 127, p. 288-293.

41. Brosgart CL, TA Louis, DW Hillman et al. Terry Beirn Community Programs for Clinical Research on AIDS: A randomized, placebo-controlled trial of the safety and efficacy of oral gancyclovir for prophylaxis of cytomegalovirus disease in HIV-infected individuals, AIDS, 1998, n 12, p. 269277.

42. Rose DN, et HS Sacks. Costeffectiveness of cytomegalovirus disease prevention in patients with AIDS: oral gancyclovir and CMV polymerase chain reaction testing, AIDS, 1997, $\mathrm{n}^{\circ} 11$, p. $883-887$.

43. Jabs DA, C Enger et JG Bartlett. Cytomegalovirus retinitis and acquired immunodeficiency syndrome, Arch Ophthalmol, 1989, no 107 , p. $75-80$.

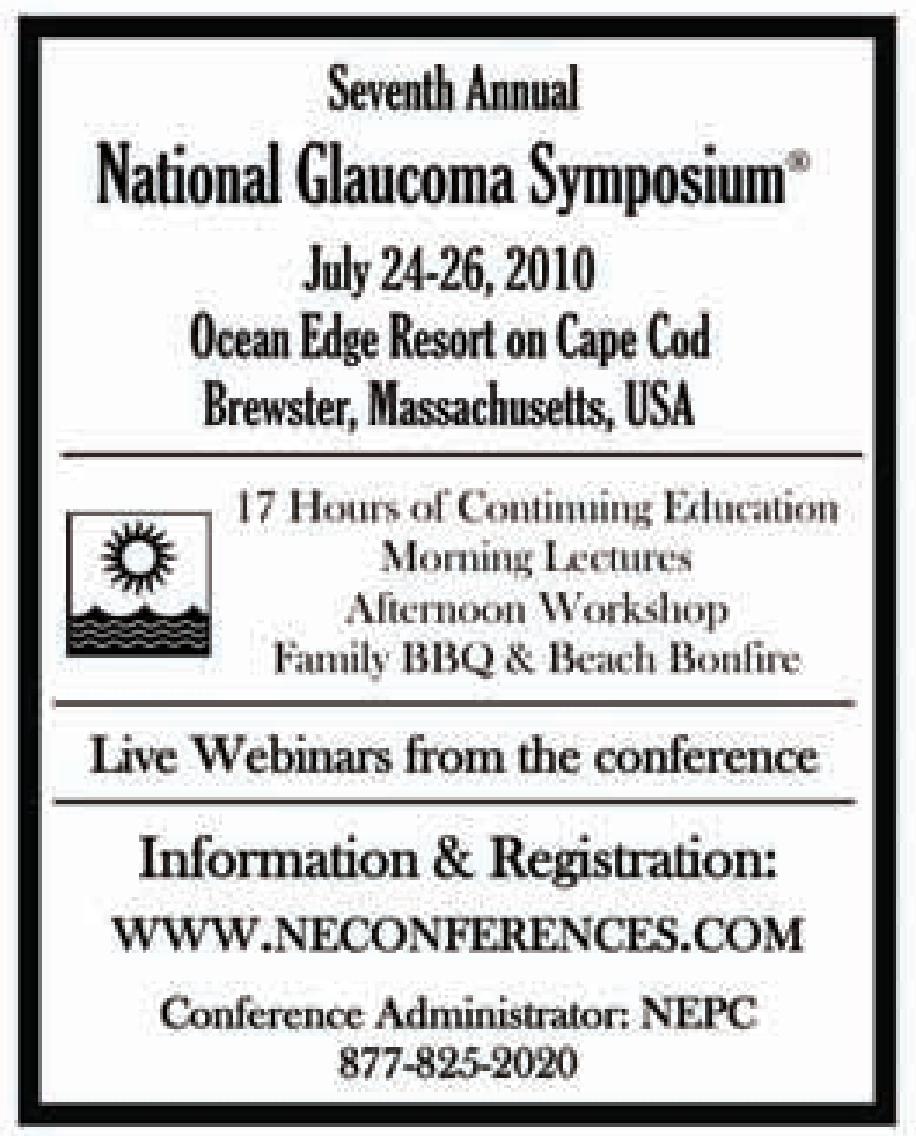

